\title{
La problématique des étiages
}

\author{
J.F. Mignot \\ EPALA \\ J. Lefèvre \\ Agence de l'Eau Loire Bretagne
}

\section{IES GRANDS ETESS EN FRANCE DEPUIS LE XII ̀̀me SIECLE}

L'analyse des étés secs ou très chauds sur une très longue période fait ressortir que l'on relève en moyenne une année chaude ou sèche tous les 5 à 6 ans (153 sur 861 ans entre 1135 et 1996).

C'est donc bien à partir d'une fréquence quinquennale que l'on ressent la notion de sécheresse.

Quelques exemples :

1135 Il fait si chaud que la terre brûlait en divers endroits de la France

1303 Année exceptionnellement sèche. La Seine, l'Oise et la Loire sont à sec....

1473 L'année s'étend à presque toute la terre.

1540 l'Année de la soilé (sécheresse)

1681 La sécheresse fut si grande dans toute la France qu'il n'y eut point de diocèse où les évêques et chapitres n'ordonnassent des prières publiques et des processions générales pour la conservation des biens de la terre

1719 Pas de pluie dans le Centre du 25 mars au $1^{\text {er }}$ novembre (sauf un orage le 24 juillet)

1785 Grande sécheresse dans toute la France

1842 Seulement $393 \mathrm{~mm}$ à Paris. Le niveau de la Seine est au plus bas

1921 Année de sécheresse sans précédent dans les bassins de la Seine et de la Loire inférieure

1949 On traverse la Loire à pied sec

.1.

S'il est assez difficile de situer l'année 1949 parmi les 800 dernières années; il est certain qu'il s'agit de l'année la plus sèche depuis une centaine d'années (fréquence environ cinquantenale ou centennale), la période 1945 - 1952 représentant une longue période de sécheresse exceptionnelle nettement plus sévère que les périodes récentes de sécheresse.

On imagine les conséquences économiques qu'elle aurait pu avoir dans les années (19)90 malgré le soutien des étiages de la Loire.
Dans un passé plus éloigné, on peut supposer que le XVIII'me siècle comporta de nombreux étés secs puisque l'on ne dénombre pas moins de 40 années où on enregistra de nombreux problèmes de sécheresse ou de chaleur (même si le résultat en fut bénéfique, comme cette année 1798 où le Médoc fut de qualité merveilleuse).

\section{RÉFLEXIONS SUR LES MÉTHODES POUR LIMITER LES CONSÉQUENCES DES SÉCHERESSES}

Le SDAGE représente une base de travail intéressante dans ce domaine en fournissant à la fois des contraintes réglementaires et une méthodologie d'approche de ces problèmes.

La loi impose que le SDAGE définisse de manière générale et harmonisée des objectifs de qualité et de quantité pour les eaux.

Pour satisfaire à cette obligation, les orientations générales prévoient que des objectifs de débit devront être fixés à l'aval des ouvrages et en certains points nodaux du bassin, et que des objectifs de qualité seront à respecter pour des tronçons de cours d'eau et en certains points nodaux du bassin. 86 points nodaux ont été définis sur le bassin Loire Bretagne (dont un certain nombre tout au long de la Loire).

La fixation d'objectifs aux points nodaux est la conséquence de choix d'usages ou de fonctions pour les cours d'eau concernés. Ces choix ont été soumis au Comité de Bassin sous la forme d'objectifs globaux pour les bassins versants des grands cours d'eau concernés par plusieurs points nodaux et sous la forme de scénarii par bassin versant limité par un point nodal.

En ce qui concerne les objectifs de débit, concernés par ce propos, trois notions ont été établies:

- le DOE (débit objectif d'étiage) qui est la valeur au-dessus de laquelle il est considéré qu'à l'aval du point nodal l'ensemble des usages est possible en équilibre avec le bon fonctionnement du milieu aquatique.

C'est un objectif structurel qui prend en compte le développement des usages. Il servira de référence à la police des 
eaux pour accorder les autorisations de prélèvements et de rejet.

Cet objectif est assorti d'un risque quinquennal et c'est d'ailleurs le débit moyen mensuel minimal de fréquence quinquennale qui sert de base à son estimation.

Il peut être, bien entendu, obtenu naturellement mais aussi par des programmes d'économies d'eau ou encore par un développement de ressources nouvelles.

Sur le bassin Loire Bretagne, les DOE résultent des principes suivants:

* Solidarité de bassin : on ne doit pas créer une gêne sur un tronçon pour permettre un développement sur un autre tronçon.

* Recherche prioritaire de la réduction des difficultés existant dans les tronçons du cours d'eau avant d'envisager un développement des usages.

* Recherche de l'amélioration du bilan en eau avant d'envisager de soutenir les étiages. Dans ce cas, le DOE est supérieur au débit de référence : c'est un lieu prioritaire de mise en oeuvre d'un SAGE.

- le DSA (débit seuil d'alerte) qui déclenche les premières mesures de restriction pour certaines activités, à l'initiative de l'autorité préfectorale.

En dessous de ce seuil, l'une des fonctions du milieu ou activité est compromise.
- le DCR (débit de crise) qui est la valeur en dessous de laquelle on considère que l'alimentation en eau potable pour les besoins indispensables à la vie humaine et animale ainsi que la survie des espèces les plus intéressantes du milieu ne sont plus garanties.

A ce niveau, toutes les mesures possibles de restriction des consommations et des rejets doivent avoir été mises en oeuvre.

En ce qui concerne la Loire, l'Allier, le Cher et la Vienne, ces objectifs sont rassemblés dans le tableau 1.

\section{III - LES ÉCONOMIES D'EAU : POSSIBILITÉS ET LIMITES}

Lorsqu'on parle de manque d'eau, il faut déterminer les causes de cette pénurie : est-elle due à un manque réel parce que les besoins ont de loin dépassé l'offre de la ressource ou bien est-elle due à une mauvaise qualité de la ressource qui, de fait, équivaut à une pénurie. Cependant le second cas devra être résolu en priorité et pour le long terme par une restauration de cette qualité même si temporairement d'autres solutions devront être adaptées.

Sinon, les économies pourront être effectuées à plusieurs niveaux :

\begin{tabular}{|c|c|c|c|c|c|}
\hline Rivière & Lieu & DOE $\left(\mathrm{m}^{3} / \mathrm{s}\right)$ & DSA $\left(\mathrm{m}^{3} / \mathrm{s}\right)$ & $\operatorname{DCR}\left(\mathrm{m}^{3} / \mathrm{s}\right)$ & QMNA5 \\
\hline \multirow[t]{9}{*}{ Loire } & Montjean & 127 & 100 & & 127 \\
\hline & & 127 & 100 & & 127 \\
\hline & & 84 & 57 & 54 & 85.5 \\
\hline & & 65 & 50 & 46 & 67.9 \\
\hline & & 60 & 50 & 48 & 63 \\
\hline & & 53 & 50 & 42 & 56 \\
\hline & & 10 & 8 & 7.5 & 9.6 \\
\hline & & 5.2 & 5 & 4.5 & 5.2 \\
\hline & & 2.3 & 2 & 1.8 & 2.3 \\
\hline \multirow[t]{8}{*}{ Allier } & & 23 & 17 & 15.5 & 23 \\
\hline & & $17.5 / 16.5$ & 13 & 12 & 17.6 \\
\hline & & 14 & 10 & 9 & 13.5 \\
\hline & & $14 / 11$ & 10 & 9 & 13.5 \\
\hline & & $14 / 11$ & 10 & 8 & 12.8 \\
\hline & & $8 / 7$ & 6 & 5.5 & 7.212 .8 \\
\hline & & 6 & 5.5 & 3 & 4 \\
\hline & & 3.2 & 3 & 2 & 2.4 \\
\hline \multirow[t]{4}{*}{ Cher } & & 18 & 7.1 & 5.2 & 16.9 \\
\hline & & 8 & 7 & 3.6 & 4.8 \\
\hline & & 5.9 & 4.9 & 1.6 & 2.8 \\
\hline & & 4 & 3 & 0.8 & 1 \\
\hline \multirow[t]{5}{*}{ Vienne } & & 32 & 25 & & 32 \\
\hline & & 20 & 16 & & 20 \\
\hline & & $12 / 10$ & 10 & & 12 \\
\hline & & 12 & 10 & & 11 \\
\hline & & 9.5 & 8 & & 8.7 \\
\hline
\end{tabular}

Tableau 1 - Objectifs de débits en période d'étiage sur la Loire, l'Allier, le Cher et la Vienne. 
* économies d'eau chez les particuliers : des expériences intéressantes sont actuellement menées en Bretagne;

* réduction de fuites sur un réseau de distribution d'eau : c'est le cas de réseaux très étendus ou encore de vieux réseaux (il n'est pas si rare d'avoir des rendements de 50 à $60 \%$ );

* économies d'eau chez les industriels : beaucoup de recyclages ont déjà été réalisés et il est probable que globalement on soit proche de l'optimum;

* économies d'eau en irrigation : c'est probablement sur ce poste qu'il y a le plus d'économies à réaliser sans toutefois se masquer les difficultés de réalisation.

On peut de suite noter que l'irrigation représente le volume de consommation le plus important du bassin (environ $45 \%$ de la consommation, qui est de plus concentrée sur quelques mois).

Les mesures consistent en équipements de compteur (objectif de connaissance et de meilleure maîtrise des arrosages), redevances majorées en zones intensément exploitées, conseils en irrigation (se rapprocher de l'optimum rendement / consommation d'eau, ou encore changement de cultures)... Cependant nous entrons alors dans un domaine économique où l'agriculteur n'est plus seul maître du jeu (marché mondial, politique $\mathrm{PAC}, \ldots$ ).

Ce sont globalement ces types de mesures qui sont à mettre en cuvre ou tout au moins à prendre en compte avant de se lancer dans des solution d'aménagement qui en général font appel au financement public de manière extrêmement importante. Cependant dans certaines régions, notamment karstiques, elles sont pratiquement les seules à pouvoir être mises en oeuvre.

\section{1 LES AMÉLIORATIONS ET LES CRÉATIONS DE RESSOURCE EN EAU}

Nous n'insisterons pas beaucoup sur ce domaine bien connu qui a fait partie des solutions d'aménagement retenues a priori pendant toute la période d'expansion économique.

Il faut cependant noter que désormais tous les projets doivent avoir été justifiés tant sur le plan technique qu'économique. Il n'est plus nécessaire de réaliser un barrage jusqu'à la capacité maximale du site, de créer un volume pour pallier les déficiences d'approvisionnement pour l'année centennale ou millénale. De même les études d'impact prennent aujourd'hui en compte des aspects nouveaux.

Par ailleurs, avant de songer à créer des retenues qui ont des impacts importants sur l'environnement, il faut regarder d'autres solutions qui peuvent résoudre le problème dans d'aussi bonnes conditions :

* les transferts d'eau: sans aller jusqu'au projet Rhône Loire (une autre solution au soutien des étiages), c'est une solution «couramment» adoptée dans les régions les plus sèches du bassin (Bretagne, Vendée)

* les recharges de nappe qui se sont peu développées jusqu'à présent dans le bassin Loire Bretagne. Il faut dire qu'elles réclament des conditions géologiques particulières. 


\section{BON DE COMMANDE :}

NOM :

ORGANISME :

ADRESSE :

\section{JOURNEES DE L'HYDRAULIQUE :}

1 lèmes. Utilisation des ressources en eau d'un bassin dans le cadre de l'aménagement du territoire (1970) 12 èmes. Hydrotechnique des liquides industriels (1972)

13èmes Influence des activités de l'homme sur le cycle hydrométéorologique (1974) ............................. $300 \mathrm{~F}$

14èmes. La mécanique des Fluides et l'environnement (1976) ........................................................... $300 \mathrm{~F}$

15èmes. L'Hydrotechnique au service d'une politique de l'Eau (1978) ................................................. $300 \mathrm{~F}$

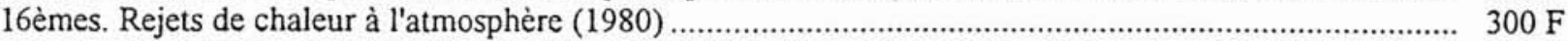

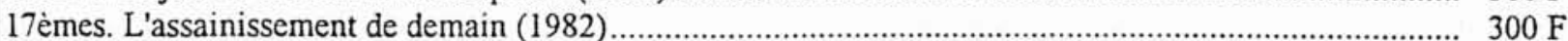

18èmes. L'Hydraulique et la maîtrise du Littoral (1984) ............................................................. $300 \mathrm{~F}$

19èmes. L'Impact des activité humaines sur les eaux continentales (1986)................................................ épuisé

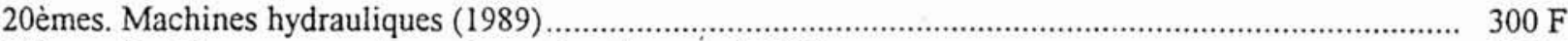

21èmes. Les Eaux souterraines et la gestion des eaux (1991) .............................................................. $300 \mathrm{~F}$

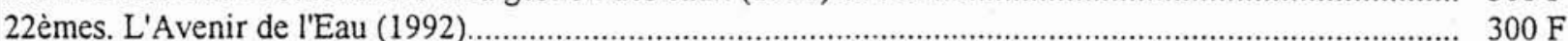

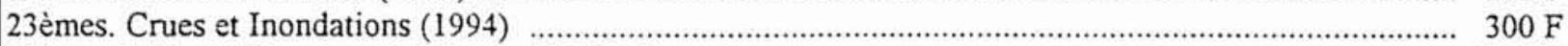

24èmes L'Eau, l'Homme et la Nature (1996) à paraître ................................................................... $1000 \mathrm{~F}$ COLLOQUES D'HYDROTECHNIQUE :

Les Sécheresses (1989).

Les modèles mathématiques pour la gestion de la qualité des eaux superficielles (1989)

Economie de l'hydrologie urbaine (1990)

Formation de l'Ingénieur hydraulicien européen (1991).

Stockage de l'eau et maîtrise des débits (1991)

Cavitation (1992)

Irrigation 2000 (1992)......

Crues at Laves torrentielles (1992)

La pluie, source de vie, choc de pollution (1993).

Bruit et Vibrations des Pompes (1993)

Transports solides en eaux continentale et littorale (1993).

Mesure et contrôle de débits en conduite (1994)

Eaux minérales et thermales (1994)

Glaciologie et nivologie - Etat des recherches et des connaissances à la fin du XXè s.(1995)

Journée d'information sur les SDAGE (1995).

Connaissance et valorisation des nappes profondes (1995)

La Loire (1996)

\section{GUIDES :}

Prévision des crues (1987)

épuisé

èpuisé $600 \mathrm{~F}$

épuisé

épuisé

$600 \mathrm{~F}$

$200 \mathrm{~F}$

j00 F

$200 \mathrm{~F}$

épuisé

$200 \mathrm{~F}$

$200 \mathrm{~F}$

épuisé

$200 \mathrm{~F}$

$100 \mathrm{~F}$

épuisé

Conception, réalisation et exploitation des petits aménagements hydroélectriques (1985)

édition française

.édition anglaise

Législation des petites centrales - France (1991).

La Cavitation (1995) (P.U.G.)

\section{CAHIERS DES CHARGES - TYPES :}

Pour la fourniture de turbines hydrauliques :

1-turbines Francis, Pelton et Kaplan, +

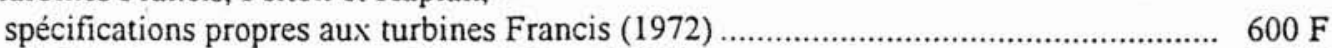

2 -spécifications propres aux turbines Pelton (1972) ............................................. $200 \mathrm{~F}$

3-spécifications propres aux turbines Kaplan (1973) ......................................... $200 \mathrm{~F}$

4-groupes du type Bulbe (1974) ..................................................................... $600 \mathrm{~F}$

édition française ou .édition anglaise

Pour la fourniture et le montage des conduites forcées en acier et de leurs accessoires (1972) .................. $800 \mathrm{~F}$

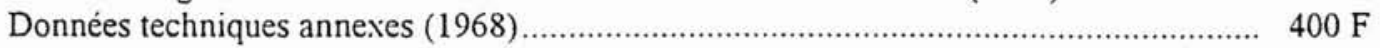

